\title{
Determination of the local tie vector between the VLBI and GNSS reference points at Onsala using GPS measurements
}

\author{
T. Ning • R. Haas • G. Elgered
}

5 the date of receipt and acceptance should be inserted later

6 Abstract Two gimbal-mounted GNSS antennas were installed on each side of the 7 radome-enclosed $20 \mathrm{~m}$ VLBI radio telescope at the Onsala Space Observatory. GPS 8 data with a $1 \mathrm{~Hz}$ sampling rate were recorded for five semi-kinematic and four kinematic observing campaigns. These GPS data were analysed together with data from the IGS station ONSA with an in-house Matlab-based GPS software package, using the double-difference analysis strategy. The coordinates of the GNSS antennas on the telescope were estimated for different observation angles of the telescope, at specific epochs, and used to calculate the geodetic reference point of the telescope. The local tie vector between the VLBI and the ONSA GNSS reference points in a geocentric reference frame was hence obtained. The two different types of observing campaigns gave consistent results of the estimated local tie vector and the axis offset of the telescope. The estimated local tie vector obtained from all nine campaigns gave standard deviations of $1.5 \mathrm{~mm}, 1.0 \mathrm{~mm}$, and $2.9 \mathrm{~mm}$ for the geocentric $\mathrm{X}, \mathrm{Y}$, and $\mathrm{Z}$ components, respectively. The result of the estimated axis offset of the VLBI telescope shows a difference of $0.3 \mathrm{~mm}$, with a standard deviation of $1.9 \mathrm{~mm}$, with respect to a reference value obtained by two local surveys carried out in 2002 and 2008. Our results show that the presented method can be used as a complement to the more accurate but more labour intensive classical geodetic surveys to continuously monitor the local tie at co-location stations with an accuracy of a few millimetres.

Keywords VLBI radio telescope - geodetic reference point · axis offset · GNSS .

GPS $\cdot$ local tie vector

T. Ning $\cdot$ R. Haas $\cdot$ G. Elgered

Department of Earth and Space Sciences, Chalmers University of Technology, Onsala Space Observatory, SE-43992 Onsala.

E-mail: tong.ning/rudiger.haas/gunnar.elgered@chalmers.se

T. Ning

GeoForschungsZentrum Potsdam, Telegrafenberg A17, Potsdam 14473, Germany

E-mail: tongn@gfz-potsdam.de 


\section{Introduction}

The International Terrestrial Reference System (ITRS) is a global reference system which co-rotates with the Earth in its diurnal motion in space (IERS, 2005). An International Terrestrial Reference Frame (ITRF) is a realization of the ITRS. ITRS coordinates are obtained using observations from space geodesy techniques (Altamimi et al., 2001), such as Global Navigation Satellite Systems (GNSS), e.g. the Global Positioning System (GPS), and Very Long Baseline Interferometry (VLBI). The Onsala Space Observatory (OSO), located at the west coast of Sweden, has been contributing to the ITRF over three decades acquiring VLBI and GPS observations. Therefore, the local tie vector between the VLBI and the GNSS reference points at Onsala and its potential change over time are of major importance for the maintenance of the ITRF.

In order to measure the local tie vector between the VLBI and the GNSS reference points, we need to determine the invariant point (IVP) of the VLBI telescope. The IVP is the intersection of the primary axis with the shortest vector between the primary azimuth and the secondary elevation axis (Dawson et al., 2007). For the telescope used in geodetic VLBI at OSO, the IVP does not exist as a physical point. Additionally, the primary and the secondary axis are not intersecting. Therefore, the IVP is the projection of the secondary axis on the primary axis indicating that the IVP can only be measured by indirect surveying methods (Eschelbach and Haas, 2005).

The determination of the invariant point of the OSO $20 \mathrm{~m}$ telescope is complicated because that this telescope is enclosed by a protecting radome of $30 \mathrm{~m}$ diameter. In 2002 a classical geodetic measurement was carried out at OSO at two different epochs. For the first epoch several survey markers were installed inside the radome to determine the endpoints of the elevation axis for different azimuth directions and for the second epoch magnetic survey markers were installed on the telescope cabin that acted as synthetic elevation axis endpoints. Successively the reference point of the radio telescope was determined by $3 \mathrm{D}$ circle fitting to the elevation axis endpoints (Eschelbach and Haas, 2005). The standard deviations of the resulting reference points coordinates were below $0.3 \mathrm{~mm}$ for both epochs and the local tie vector between the VLBI and the GNSS reference points was determined at the sub-millimetre level.

In 2008 another geodetic measurement was performed with a laser tracker. This instrument is also capable of providing local tie results at the sub-millimetre level (Lösler, 2009). In the 2008 campaign, the baseline between the IVS site (Onsala) and the IGS site (ONSA) was also measured and compared to the one obtained from the 2002 campaign. The measured baseline between the IVS and the IGS reference points in 2002 and 2008 are $79.5685 \mathrm{~m}$ and $79.5678 \mathrm{~m}$, respectively. Although the accuracy of the resulting local tie vector is high, the invested time for performing the classical measurements was many days and the procedure of the measurements is usually laborious.

One idea to avoid labor-intensive classical geodetic surveys for the determination of radio telescope invariant points and local ties, is to use GNSS. In their pioneering work, Combrinck and Merry (1997) describe a project where one gimbal-mounted GNSS antenna on the Hartebeesthoek $26 \mathrm{~m}$ radio telescope was used for the determination of the telescope's invariant point and axis offset. However, Combrinck and Merry (1997) did not apply corrections for GNSS antenna phase centre variations. 
Combrinck and Merry (1997) performed a two-step analysis involving circle-fitting analyses and did not give information on the repeatability of their results.

Also Abbondanza et al. (2009) used GNSS for local tie measurements. They performed campaigns in 2002 and 2006 with two gimbal-mounted GNSS antennas on the Medicina $32 \mathrm{~m}$ radio telescope. These campaigns were performed semikinematically and the data were analyzed with a commercial GPS analysis software, followed by post-processing to derive local tie information. Corrections for antenna phase centre variations were applied in their processing.

Kallio and Poutanen (2012) were the first to use gimbal-mounted GNSS antennas on a radome-enclosed radio telescope. They mounted two GNSS antennnas on the Metsähovi $14 \mathrm{~m}$ radio telescope and performed several kinematic observing sessions during VLBI observations to determine the local tie at Metsähovi. Based on a model first presented by Lösler (2009), they proposed a modified model where the telescope axes can be presented in the same three dimensional Cartesian system as the observed coordinates. This is well suited to measurements obtained by the GNSS antennas that are attached to the telescope structure. Kallio and Poutanen (2012) used a two-step approach for the local tie determination which consisted of the actual GPS data analysis with a commercial software and a post-processing step. In their analysis they consider corrections of antenna phase centre variations. However, to the authors knowledge non of these studies took into consideration that the gimbal-mounted antenna on the telescope experience different hydrostatic delays when the telescope was pointed at different elevation angles.

Inspired by the work of Kallio and Poutanen (2012), two gimbal-mounted GNSS antennas were installed on the $20 \mathrm{~m}$ radome-enclosed VLBI radio telescope at OSO in the summer of 2013, one on each side of the main reflector. Thereafter, GPS data were recorded during several campaigns, both semi-kinematic and kinematic ones, and the coordinates of the GNSS antennas were determined to estimate both the local tie vector between the VLBI and the GNSS reference points and the axis offset of the telescope. Section 2 describes the models and the rotation matrices which were used in order to transform the estimated GPS coordinates to the IVP of the telescope. A prerequisite for obtaining high accuracy in the estimated GPS coordinates is to fix carrier phase ambiguities to integers. Therefore, we used double-difference carrier phase measurements in the GPS data processing, which is discussed in Section 3. In this section we also describe how the hydrostatic delay differences were treated in the analysis. The results of the estimated local tie vector and the axis offset of the telescope are presented in Section 4, followed by the conclusions and suggestions for future work in Section 5.

\section{Methodology}

We used a model developed for the Metsähovi telescope in order to calculate the IVP of the VLBI telescope from the time series of estimated GPS coordinates (Kallio and Poutanen, 2012):

$$
X_{n}=X_{0}+R_{\alpha, a}\left(E-X_{0}\right)+R_{\alpha, a} R_{\varepsilon, e} P_{n}
$$


where the coordinate vector of the GNSS antenna $X_{n}(n=1,2)$, in our case in a geocentric reference frame, is determined by the sum of three vectors (see Figure 1): the coordinate vector of the IVP of the telescope $X_{0}$; the axis offset vector $E-X_{0}$ rotated by the angle $\alpha$ about the azimuth axis unit vector $a$; and the vector from the eccentric point $E$ to the antenna point $P_{n}(n=1,2)$ rotated about the elevation axis unit vector $e$ by the angle $\varepsilon$ and about the azimuth axis unit vector $a$ by the angle $\alpha$. The two rotation matrices are expressed as:

$$
\begin{array}{r}
R_{\alpha, a}=\cos \alpha\left(\begin{array}{lll}
1 & 0 & 0 \\
0 & 1 & 0 \\
0 & 0 & 1
\end{array}\right)+(1- \\
\cos \alpha)\left(\begin{array}{lll}
a_{x} a_{x} & a_{x} a_{y} & a_{x} a_{z} \\
a_{x} a_{y} & a_{y} a_{y} & a_{y} a_{z} \\
a_{x} a_{z} & a_{y} a_{z} & a_{z} a_{z}
\end{array}\right) \\
+\sin \alpha\left(\begin{array}{ccc}
0 & -a_{z} & a_{y} \\
a_{z} & 0 & -a_{x} \\
-a_{y} & a_{x} & 0
\end{array}\right)
\end{array}
$$

and

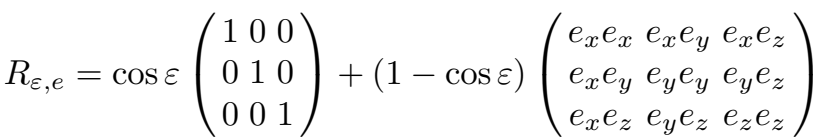

$$
\begin{aligned}
& +\sin \varepsilon\left(\begin{array}{ccc}
0 & -e_{z} & e_{y} \\
e_{z} & 0 & -e_{x} \\
-e_{y} & e_{x} & 0
\end{array}\right)
\end{aligned}
$$

In both Equations 2 and 3, there are four components for each rotation matrix: three for the axis and one for the angle. Since the axes are unit vectors, we have two condition equations, one for the azimuth axis unit vector $a$ and one for the elevation axis unit vector $e$.

$$
a_{x}^{2}+a_{y}^{2}+a_{z}^{2}=1
$$

$$
e_{x}^{2}+e_{y}^{2}+e_{z}^{2}=1
$$

Due to the fact that the offset vector $E-X_{0}$ is perpendicular to both the azimuth and the elevation axis, we have two more condition equations:

$$
\left(E-X_{0}\right)_{x} a_{x}+\left(E-X_{0}\right)_{y} a_{y}+\left(E-X_{0}\right)_{z} a_{z}=0
$$

$$
\left(E-X_{0}\right)_{x} e_{x}+\left(E-X_{0}\right)_{y} e_{y}+\left(E-X_{0}\right)_{z} e_{z}=0
$$

The input data to Equation 1 are the geocentric coordinates of the two GNSS antennas, together with the azimuth and the elevation angles of the VLBI telescope at different epochs. All unknown parameters in Equation 1 were estimated as corrections to the a priori value by solving a least squares mixed model including all condition equations and the main function:

$$
\left(\begin{array}{l}
x \\
k
\end{array}\right)_{h}=\left(\begin{array}{cc}
\sum_{i}^{t}\left[A_{i}^{T}\left(B_{i} S_{i}^{-1} B_{i}^{T}\right)^{-1} A_{i}\right] H^{T} \\
H & 0
\end{array}\right)^{-1}\left(\begin{array}{c}
\sum_{i}^{t}\left[A_{i}^{T}\left(B_{i} S_{i}^{-1} B_{i}^{T}\right)^{-1} Y_{i}\right] \\
W
\end{array}\right)
$$


where $x_{h}$ is the correction to the a priori values of the unknown parameters after $h$ times of iteration and $k$ is the vector of Lagrange multipliers; $Y_{i}$ is the basic equation for all points at epoch $i$ with the a priori values of the parameters, which is expressed for the two GNSS antennas:

$$
Y_{i}=\left(\begin{array}{ccc}
X_{1}-X_{0}^{\prime}-R_{\alpha, a^{\prime}}\left(E-X_{0}\right)^{\prime}-R_{\alpha, a^{\prime}} & R_{\varepsilon, e^{\prime}} P_{1}^{\prime} \\
X_{2}-X_{0}^{\prime}-R_{\alpha, a^{\prime}}\left(E-X_{0}\right)^{\prime}-R_{\alpha, a^{\prime}} R_{\varepsilon, e^{\prime}} P_{2}^{\prime}
\end{array}\right)
$$

where the vectors of the estimated unknown parameters are the corrections with respect to the a priori values (indicated by a prime in Equation 9):

$$
\begin{array}{r}
{\left[\triangle X_{0 x}, \triangle X_{0 y}, \triangle X_{0 z}, \triangle\left(E-X_{0}\right)_{x}, \triangle\left(E-X_{0}\right)_{y}, \triangle\left(E-X_{0}\right)_{z}, \triangle a_{x},\right.} \\
\left.\triangle a_{y}, \triangle a_{z}, \triangle e_{x}, \triangle e_{y}, \triangle e_{z}, \triangle P_{1 x}, \triangle P_{1 y}, \triangle P_{1 z}, \triangle P_{2 x}, \triangle P_{2 y}, \triangle P_{2 z}\right]
\end{array}
$$

Solving the condition equations and differentiating with respect to the correction using Equation 10 gives us the $H$ and $W$ matrices:

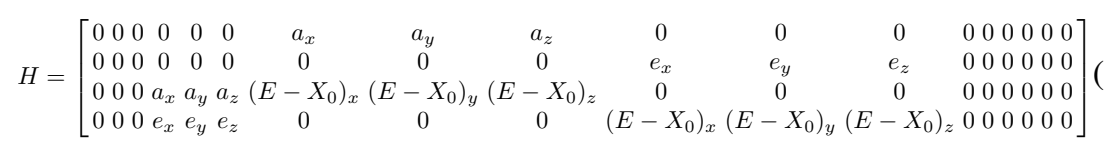

$$
W=\left[\begin{array}{c}
\frac{1}{2}\left(1-a_{x}^{2}-a_{y}^{2}-a_{z}^{2}\right) \\
\frac{1}{2}\left(1-e_{x}^{2}-e_{y}^{2}-e_{z}^{2}\right) \\
-\left(E-X_{0}\right)_{x} a_{x}-\left(E-X_{0}\right)_{y} a_{y}-\left(E-X_{0}\right)_{z} a_{z} \\
-\left(E-X_{0}\right)_{x} e_{x}-\left(E-X_{0}\right)_{y} e_{y}-\left(E-X_{0}\right)_{z} e_{z}
\end{array}\right]
$$

The partial differentiation at epoch $i$ with respect to the unknown parameters is used to construct the matrix $A_{i}$ :

$$
A_{i}=\left(\begin{array}{cccccc}
\frac{\partial Y_{i}}{\partial X_{0}} & \frac{\partial Y_{i}}{\partial\left(E-X_{0}\right)} & \frac{\partial Y_{i}}{\partial a} & \frac{\partial Y_{i}}{\partial e} & \frac{\partial Y_{i}}{\partial P_{1}} & 0 \\
\frac{\partial Y_{i}}{\partial X_{0}} & \frac{\partial Y_{i}}{\partial\left(E-X_{0}\right)} & \frac{\partial Y_{i}}{\partial a} & \frac{\partial Y_{i}}{\partial e} & 0 & \frac{\partial Y_{i}}{\partial P_{2}}
\end{array}\right)
$$

while the $B_{i}$ matrix is the partial differentiation with respect to the observations for each telescope position given at different azimuth $(A Z)$ and elevation $(E L)$ angles and for the coordinates of each GNSS antenna.

$$
B_{i}=\left(\begin{array}{cccc}
\frac{\partial Y_{i}}{\partial A Z_{i}} & \frac{\partial Y_{i}}{\partial E L_{i}} & \frac{\partial Y_{i}}{\partial X_{1}} & 0 \\
\frac{\partial Y_{i}}{\partial A Z_{i}} & \frac{\partial Y_{i}}{\partial E L_{i}} & 0 & \frac{\partial Y_{i}}{\partial X_{2}}
\end{array}\right)
$$

There is one more matrix in Equation $8, S$, which is the weighting matrix taking the uncertainty of the angle reading from the telescope and the uncertainty of the estimated coordinates from the GNSS antenna at epoch $i$ into account.

$S_{i}=\left(\begin{array}{cccccccc}\sigma_{A Z}^{2} & 0 & 0 & 0 & 0 & 0 & 0 & 0 \\ 0 & \sigma_{E L}^{2} & 0 & 0 & 0 & 0 & 0 & 0 \\ 0 & 0 & \sigma_{X_{1 x}}^{2} & \sigma_{X_{1 x}} \sigma_{X_{1 y}} & \sigma_{X_{1 x}} \sigma_{X_{1 z}} & 0 & 0 & 0 \\ 0 & 0 & \sigma_{X_{1 y}} \sigma_{X_{1 x}} & \sigma_{X_{1 y}}^{2} & \sigma_{X_{1 y}} \sigma_{X_{1 z}} & 0 & 0 & 0 \\ 0 & 0 & \sigma_{X_{1 z}} \sigma_{X_{1 x}} & \sigma_{X_{1 z}} \sigma_{X_{1 y}} & \sigma_{X_{1 z}}^{2} & 0 & 0 & 0 \\ 0 & 0 & 0 & 0 & 0 & \sigma_{X_{2 x}}^{2} & \sigma_{X_{2 x}} \sigma_{X_{2 y}} & \sigma_{X_{2 x}} \sigma_{X_{2 z}} \\ 0 & 0 & 0 & 0 & 0 & \sigma_{X_{2 y}} \sigma_{X_{2 x}} & \sigma_{X_{2 y}}^{2} & \sigma_{X_{2 y}} \sigma_{X_{2 z}} \\ 0 & 0 & 0 & 0 & 0 & \sigma_{X_{2 z}} \sigma_{X_{2 x}} \sigma_{X_{2 z}} \sigma_{X_{2 y}} & \sigma_{X_{2 z}}^{2}\end{array}\right)^{-1}$ 
The solution of Equation 8 is obtained by iterations until convergence is found. We defined convergence when the corrections to the IVP coordinates become less than $0.1 \mathrm{~mm}$.

\section{GPS observations and data processing}

Two Leica AS10 multi-GNSS antennas were mounted on both sides of the telescope dish using two rotating holders. Both holders have counterweights in order to make the two antennas point to the zenith regardless of the position of the VLBI telescope (see Figure 2). The sampling rate of the GPS measurements was $1 \mathrm{~Hz}$ and the data were recorded for two types of sessions, semi-kinematic and kinematic.

In the semi-kinematic sessions, the telescope was scheduled in a sequence of different azimuth and elevation angles. The duration of each session was 24 hours. For the first two sessions (July 9 and 10, 2013), the telescope was positioned at elevation angles $10^{\circ}, 15^{\circ}, 20^{\circ}, 25^{\circ}, 30^{\circ}, 35^{\circ}, 40^{\circ}, 45^{\circ}, 55^{\circ}, 65^{\circ}, 75^{\circ}$, and $85^{\circ}$. For each elevation angle, the telescope was positioned at four different azimuth angles with an interval of $90^{\circ}$. In total, this approach gave 48 different telescope positions. After each 30 minutes the telescope moved to a new position. For the other three semikinematic sessions (September 21-23, 2013), the telescope moved through the same elevation angles as for the first two sessions, but with four more azimuth angles for each elevation angle with an interval of $45^{\circ}$, which in total gave us 96 different telescope positions, and hence 15 minutes were spend in each direction.

During the kinematic sessions, GPS observations were recorded during four standard VLBI sessions. All sessions are summarized in Table 1.

In the data processing we only used GPS data acquired when the VLBI antenna was at the planned position (semi-kinematic sessions) or tracking the scheduled radio source (kinematic sessions). The data acquired when the telescope was moving between the fixed positions, or slewing between radio sources, were excluded. The azimuth speed of the telescope is elevation dependent when tracking a radio source. It is highest when a radio source passes through the local zenith and the telescope has to move by half a turn in azimuth to follow the source. During the four VLBI sessions used for this work, $84 \%$ of the observations were acquired at an elevation angle below $60^{\circ}$. For these observations the telescope speed in elevation and azimuth are less than $0.5 \mathrm{arcsec} / \mathrm{s}(0.03 \mathrm{~mm} / \mathrm{s})$ and less than $27 \mathrm{arcsec} / \mathrm{s}(1.6 \mathrm{~mm} / \mathrm{s})$, respectively. In order to have correct observation angles of the telescope corresponding to the actual position of the GPS antenna, we used the angle readings from the telescope $\log$ file which is updated every second, i.e. with a temporal resolution that is identical to the GPS sampling rate. The uncertainty of the angle reading is $10 \operatorname{arcsec}$ which corresponds to an uncertainty in the position of $0.5 \mathrm{~mm}$.

An absolute correction of the Phase Centre Variations (PCV) of the GNSS antenna is necessary in the GPS data processing (Schmid et al., 2007). In our case, it is complicated to implement since the azimuth orientation of the GNSS antenna changes with the azimuth pointing of the telescope. If we apply the standard absolute PCV correction directly, it would cause systematic errors in the estimated GPS coordinates and the resulting IVP of the telescope. In order to reduce this problem, 
we calculated modified PCV corrections for the two GNSS antennas, using the azimuth orientation of the telescope and applied these to the RINEX files. Eventually, the corrected RINEX files were used in the GPS data processing.

Since the horizontal distance between the two GNSS antennas (GPS1 and GPS2) on the telescope and the IGS station ONSA is around $78 \mathrm{~m}$, the received signals should experience a common ionospheric delay. We took advantage of this feature in our data processing by forming two baselines (GPS1-ONSA and GPS2-ONSA) in order to avoid the estimation of the common parameter. Since the height difference between the two GNSS antennas on the telescope and ONSA can vary between $12.7 \mathrm{~m}$ and $18.9 \mathrm{~m}$ depending on the telescope elevation, the differential neutral atmospheric delay can be ignored only for the wet part (Ning et al., 2012) while a compensation for the hydrostatic delay was necessary (Snajdrova et al., 2005). Figure 3 depicts an example of the difference of the Zenith Hydrostatic Delay (ZHD) due to the height difference between the two GNSS antennas on the telescope and ONSA for 12 elevations of the telescope (obtained from one of the semi-kinematic sessions). In order to determine the height difference, the GPS data were first processed without corrections for the hydrostatic delay. Then the estimated height difference was used to calculate the correction for the hydrostatic delay which was then implemented in the GPS data for the final processing. We also investigated the impact of the error in the determination of the height difference on the resulting GPS coordinates. The result showed that the deviation of $1 \mathrm{~m}$ in the height difference can only cause an error in the estimated vertical component less than $1 \mathrm{~mm}$ while no difference seen for the horizontal components. However, if we ignore the ZHD corrections, the difference in the estimated vertical component can be up to $10 \mathrm{~mm}$.

Since GPS measurements were acquired kinematically, especially from the four standard VLBI sessions, the GNSS antennas were only static for very short observational time spans where the ambiguities, when estimated as floats, become poorly separable from the baseline coordinates. Therefore, we used double-difference data processing, using our own in-house Matlab-based GPS software, with carrier phase ambiguities fixed to integers using the LAMBDA method (Teunissen, 1993). For the following analyses, we only used solutions where the float ambiguities could be fixed to integers. In addition, we took the geometry of the satellite constellation into account by only accepting solutions when the position dilution of precision (PDOP) value was less than 5. Figure 4 demonstrates the number of GPS solutions together with the corresponding length of the observing time while the telescope was tracking on a target for two kinematic sessions: R1591 and RV101. The length of observing time for each target varies approximately from $50 \mathrm{~s}$ to $500 \mathrm{~s}$. It is evident that for the telescope positions with very short observing time, i.e. less than $50 \mathrm{~s}$, no solutions were given by both GPS1 and GPS2. This is because that the duration time is too short for an ambiguity resolution. For some telescope positions, with longer duration time, we see solutions only from one of the GPS antennas. It indicates the impact of the telescope itself blocking the incoming signals from GPS satellites.

In order to reject outliers in the estimated coordinates after the GPS data processing, we used the distance and the height difference between the two GNSS antennas, GPS1 and GPS2, as references. The expected distance and the height difference between GPS1 and GPS2 were estimated by the GPS data acquired from two static 
sessions (July 6-7, 2013) where the telescope was static and pointing to the zenith. The deviations of the estimated distance and height difference, given by the time series of estimated coordinates of GPS1 and GPS2, during all nine sessions, from the expected value were examined for outlier detection. All data points with a difference from the expected distance $(24.749 \mathrm{~m})$ larger than one standard deviation were removed while we excluded the data points with a height difference deviating from the expected value $(0.005 \mathrm{~m})$ more than $0.1 \mathrm{~m}$. Table 1 shows the number of data points (epochs) after the GPS data processing (Step 1) and after the outlier detection (Step 2) for each session. For most sessions, around $55 \%$ of data points were excluded as outliers while more data points ( $78 \%)$ were excluded for one VLBI session (RV101) having more short observations.

After the outlier detection, the GPS coordinates and the corresponding telescope angle reading (azimuth and elevation) were used for the linearized least squares mixed model with condition equations (see Equations 1 to 7). After the first two iterations, the data points with residuals larger than $50 \mathrm{~cm}$ were removed and after another two iterations, the threshold value was set to $25 \mathrm{~cm}$. Then, after two more iterations, the data points with residuals larger than three standard deviations were removed. Thereafter, we iterated the analysis until convergence was reached. Table 1 shows the number of data points included in the last iteration (Step 3). For most sessions, over $94 \%$ of the input data to the model were included in the final stage. This indicates that most bad data points were excluded by our outlier detection based on the distance and the height difference between GPS1 and GPS2. Table 1 also shows the total number of telescope positions (Step 0) for each session, the number of telescope positions left after the GPS data processing and after the outlier exclusion, as well as the number of telescope positions included in the last iteration. For the semi-kinematic sessions, around $60 \%$ of the telescope positions were used in the final estimation where most of the position rejection occurred in the GPS data processing due to the failure of fixing ambiguities to integers. For the kinematic sessions, many more telescope positions were excluded (only $5 \%$ to $22 \%$ positions were left in the final stage) where approximately half of the rejections happened during the GPS data processing while the other half was due to the outlier exclusion.

\section{Results}

The estimated local tie vector, in a geocentric reference frame, between the VLBI and the GNSS reference points, together with the estimated axis offset of the telescope, as well as the $P$ vectors (the vector from the eccentric point to the reference point of the GNSS antenna) are given in Table 2, while the corresponding covariance matrix of the local tie vector is given in Table 3 . The coordinates of the IGS station ONSA were given by the data processing using GIPSY/OASIS II v.6.2 (Webb and Zumberge, 1993) with the Precise Point Positioning (PPP) strategy (Zumberge et al., 1997). We have results from the semi-kinematic sessions for five days and for four days from the kinematic sessions. The results show no significant difference between the two approaches in terms of mean values while the semi-kinematic approach have slightly lower standard deviations. If we convert the local tie vector to topocentric 
coordinates (shown in Table 4), a larger standard deviation of $4.9 \mathrm{~mm}$ is seen for the vertical component from the kinematic sessions.

The mean baseline, in Table 2, given by the semi-kinematic sessions is $79.5744 \mathrm{~m}$ with a standard deviation of $1.1 \mathrm{~mm}$ while the ones for the kinematic sessions are $79.5738 \mathrm{~m}$ and $1.3 \mathrm{~mm}$, respectively. The axis offset, given by the semi-kinematic sessions, is $-6.1 \mathrm{~mm}$ with a standard deviation of $1.9 \mathrm{~mm}$ while the one given by the kinematic sessions is $-6.4 \mathrm{~mm}$ with a standard deviation of $1.9 \mathrm{~mm}$. A difference within $0.5 \mathrm{~mm}$ is seen with respect to the axis offset measured by two local surveys $(-6.0 \pm 0.4 \mathrm{~mm}$ for 2002 (Eschelbach and Haas, 2005) and $-6.2 \pm 0.2 \mathrm{~mm}$ for 2008 (Lösler and Haas, 2009)). The absolute vector differences for both the $\mathrm{P}_{1}$ and the $\mathrm{P}_{2}$ vectors are below $3 \mathrm{~mm}$ when comparing the values obtained from the two types of sessions.

Table 2 also gives the combined results from all nine sessions where the standard deviations for the $\mathrm{X}, \mathrm{Y}$, and $\mathrm{Z}$ axis are $1.5 \mathrm{~mm}, 1.0 \mathrm{~mm}$ and $2.9 \mathrm{~mm}$, respectively. The estimated axis offset of the telescope shows a difference of $0.5 \mathrm{~mm}$ from the reference axis offset given by two local surveys while a standard deviation of $2.9 \mathrm{~mm}$ is seen over all sessions. For a comparison, we calculated the local tie vector in ITRF2008 (Altamimi et al., 2011) coordinates referring to the epoch of July 1, 2013. The $\mathrm{Y}$ axis shows the smallest difference $(-1.2 \mathrm{~mm})$ from the ITRF value, while the differences for the $\mathrm{X}$ and $\mathrm{Z}$ axis are $2.0 \mathrm{~mm}$ and $5.0 \mathrm{~mm}$, respectively. A difference of $3.3 \mathrm{~mm}$ is seen between the estimated baseline and the ITRF baseline. Some parts of the difference is due to the influence of thermal effects on the telescope structure (Lösler et al., 2013). The height difference due to the temperature difference can be modelled by Equation 15 presented by Lösler et al. (2013). Based on local meteorological observations, the mean ground temperature for all nine sessions are $15{ }^{\circ} \mathrm{C}$. If we take the thermal deformation of the telescope into account and refer all results to a temperature of $0{ }^{\circ} \mathrm{C}$ (Lösler and Haas, 2009), the difference of the baseline is reduced to $2.8 \mathrm{~mm}$. These discrepancies are on the same order of magnitude as found during the preparation of ITRF2008 (Altamimi et al., 2011), though the discrepancies are not identical per coordinate component. The rest of the difference is likely to be explained by the uncertainties in the GPS measurements which are caused by multipath effects and by the errors in the phase centre correction (PCC) due to differences between the GPS antenna correction models. In this work, the two Leica AS10, GPS1 and GPS2 on the telescope, were sent to the University of Bonn for individual calibration. Thereafter, only the model provided by the individual calibration were used. For the IGS site ONSA, however, we implemented the model given in igs08.atx which provides a mean value of the calibrations from the same type of antennas. The position offsets resulting from the use of individual calibrations and the mean calibration from igs08.atx were investigated by Baire et al. (2013). They found the position offsets for the horizontal and vertical components can be as large as $4 \mathrm{~mm}$ and $10 \mathrm{~mm}$, respectively. Furthermore, the ONSA antenna is covered by an uncalibrated plastic radome, which can cause effects primarily on the vertical component with the order of a couple of millimetres. Such effects were investigated by Ning et al. (2011) where a deviation of the order of a couple of millimetres on the vertical component was found. They also found the size of this vertical deviation varied as- 
sociated with different geometries of the electromagnetic environment of the antenna as well as with the elevation cutoff angle for the observations used in the analysis.

Since the distance between the two GNSS antennas on the telescope is fixed, we could take this fixed baseline as a condition for our GPS data processing. We combined the relative coordinates of GPS1-ONSA and GPS1-ONSA from previous data processing and used them as a priori coordinates. The corrections for the a priori coordinates were obtained by solving a least squares model again and fixing the baseline between GPS1 and GPS2. The differences in the estimated local tie given by the GPS data processing using a non-fixed and fixed baseline are shown in Figure 5. No significant changes, in terms of both the mean and standard deviation, are seen for the estimated relative coordinates after we fixed the baseline while the non-fixed solution actually gives a better result in the estimated axis offset.

We know the axis offset of the telescope, with a sub-mm accuracy, from the two local surveys performed in 2002 and 2008. We thus can fix the axis offset value in our least squares mixed model in order to reduce number of unknown parameters. Figure 6 depicts the estimated local tie vector with and without fixing the axis offset value. An insignificant difference $(<1 \mathrm{~mm})$ is observed in the results obtained with and without fixing the axis offset.

As discussed earlier, the orientation of the GNSS antenna on the telescope varies with the azimuth pointing of the telescope, meaning that direct implementation of the standard absolute PCV corrections will cause systematic errors in the estimated local tie vector. This is depicted by Figure 7 where the blue squares show the results given by the GPS data processing with the direct implementation of the standard absolute PCV corrections and the red circles show the results using the modified PCV corrections. Clear systematic offsets are seen for the results using the standard absolute PCV corrections for the two GNSS antennas. Averaged over all nine sessions, the offsets are $0.3 \mathrm{~mm}$ for the $\mathrm{X}$ axis, $2.9 \mathrm{~mm}$ for the $\mathrm{Y}$ axis, and $1.6 \mathrm{~mm}$ for the $\mathrm{Z}$ axis, respectively while the offset for the axis offset is $1.5 \mathrm{~mm}$. This indicates that in spite of the poor electromagnetic environment PCV corrections are important and shall be applied to improve the accuracy.

\section{Conclusions and future work}

We carried out five semi-kinematic and four kinematic observing sessions with the two GNSS antennas mounted on the rim of the main reflector of the Onsala $20 \mathrm{~m}$ radio telescope. The telescope was pointed in different azimuth and elevation angles and the resulting coordinates of the two GNSS antennas were used to determine the telescope invariant point and the local tie vector between the VLBI and the GNSS reference points directly in a geocentric reference frame.

The result shows no significant differences in the estimated local tie vector and the axis offset of the telescope obtained from the two approaches. After combination of the results from all nine sessions, the differences between our estimated local tie vector and the one of ITRF2008 are $2.0 \mathrm{~mm}$ for the $\mathrm{X}$ axis, $-1.2 \mathrm{~mm}$ for the $\mathrm{Y}$ axis, and $5.0 \mathrm{~mm}$ for the $\mathrm{Z}$ axis. The smallest standard deviation of $1.0 \mathrm{~mm}$ is seen for the $\mathrm{Y}$ axis while the standard deviations for the $\mathrm{X}$ and $\mathrm{Z}$ axis are $1.5 \mathrm{~mm}$ and $2.9 \mathrm{~mm}$, 
respectively. A difference of $3.3 \mathrm{~mm}$ is seen between our estimated baseline and the ITRF2008 baseline. Part of the difference is due to the influence of thermal effects on the telescope structure while the others are likely to be explained by the uncertainties in GPS measurements caused by multipath effects, the differences in GPS antenna calibration models, and the uncalibrated plastic radome. The discrepancies are on the same order of magnitude as found during the preparation of ITRF2008 (Altamimi et al., 2011) where the local tie information by Lösler and Haas (2009) based on classical measurements were used. Systematic studies are necessary to investigate the reason for these discrepancies, in particular in the $\mathrm{Z}$ axis, using individual calibration for all GNSS antennas. In the future, GPS observation sessions for a longer time period, e.g. over one month, are desired in order to reduce the impact of the uncertainty from the vertical component of the GPS coordinates.

Due to the blockage by the telescope, a significant number of cycle slips occurred in the GPS phase measurements which introduces additional ambiguity parameters. Therefore, a higher sampling rate of GPS measurements, e.g. $10 \mathrm{~Hz}$ or $20 \mathrm{~Hz}$, would be beneficial in order to have more data available for the ambiguity estimation.

We have shown that the method can be applied not only for dedicated semikinematic campaigns but also during normal geodetic VLBI experiments. This means that this method allows to continually monitor the local tie at a station, which is of interest in particular for the co-location stations that will contribute to the upcoming VLBI Global Observing System (VGOS) operations of the IVS, like the Onsala Space Observatory.

\section{References}

Abbondanza C, Altamimi Z, Sarti P, Negusini M, Vittuari L (2009) Local effects of redundant terrestrial and GPS-based tie vectors in ITRF-like combinations, J. Geod., 83:1031-1040, doi: 10.1007/s00190-009-0321-6.

Altamimi Z, Angermann D, Argus D, Boucher C, Chao B, Drewes H, Eanes R, Feissel M, Ferland R, Herring T, Holt B, Johannson J, Larson C, Ma C, Manning J, Meertens C, Nothnagel A, Pavlis E, Petit G, Ray J, Ries J, Scherneck H.-G, Sillard P, Watkins M (2001) The Terrestrial Reference Frame and the Dynamic Earth, EOS, Transactions, 82(25), 275-278.

Altamimi Z, Collilieux X, Métivier L (2011), ITRF2008: an improved solution of the international terrestrial reference frame, J. Geod., 85, 457-473, doi: 10.1007/s00190-011-0444-4.

Baire Q, Bruyninx C, Legrand J, Pottiaux E, Aerts W, Defraigne P, Bergeot N, Chevalier JM (2013), Influence of different GPS receiver antenna calibration models on geodetic positioning, GPS Solut., 18(4), 529-539, doi: 10.1007/s10291-013-03491.

Boehm J, Werl B, Schuh H (2006) Troposphere mapping functions for GPS and very long baseline interferometry from European Centre for Medium-Range Weather Forecasts operational analysis data, J. Geophys. Res., 111, B02406, doi:10.1029/2005JB003629. 
Combrinck W L, Merry C L (1997) Very long baseline interferometry antenna axis offset and intersection determination using GPS, J Geophys. Res., 102(B11), 24741-24743, doi:10.1029/97JB02081.

Dawson J, Sarti P, Johnston GM, Vittuari L (2007) Indirect approach to invariant point determination for SLR and VLBI systems: an assessment, J. Geod., 81(6-8), 433-441, doi:10.1007/s00190-006-0125-x.

Eschelbach C, Haas R (2005) The 2002 Local Tie Survey at the Onsala Space Observatory, In: Proc. IERS Workshop on site co-location, edited by B. Richter, W. Schwegmann and W.R. Dick, IERS Technical Note, 33, Verlag des Bundesamts für Kartographie und Geodäsie, 55-63.

IERS (2005) In: Richter B, Schwegmann W, Dick WR (eds) Proceedings of the IERS workshop on site co-location, Matera, Italy, 23-24 Oct 2003. IERS technical note No. 33. Verlag des Bundesamts für Kartographie und Geodäsie, Frankfurt am Main, p. 148.

Lösler M (2009) New mathematical model for reference point determination of an azimuth-elevation type radio telescope. J. Surv. Eng., 135(4): 131-135.

Lösler M, Haas R (2009) The 2008 Local-tie Survey at the Onsala Space Observatory, In Charlot P., A. Collioud and G. Bourda, G. (Ed.) Proceedings of the 19th European VLBI for Geodesy and Astrometry Working Meeting, March 24-25, 2009, Bordeaux, France, 97-101.

Lösler M, Haas R, Eschelbach C (2013) Automated and Continual Determination of Radio Telescope Reference Points With Sub-mm Accuracy: Results from a campaign at the Onsala Space Observatory. J. Geod., 87(8), 791-804, doi:10.1007/s00190-013-0647-y.

Ning T, Elgered G, Johansson, JM (2011), The impact of microwave absorber and radome geometries on GNSS measurements of station coordinates and atmospheric water vapour, Adv. Space. Res., 47(2), 186-196, doi:10.1016/j.asr.2010.06.023.

Ning T, Haas R, Elgered G, Willén U, (2012) Multi-Technique Comparisons of Ten Years of Wet Delay Estimates on the West Coast of Sweden, J. Geod., 86(7), 565575, doi:10.1007/s00190-011-0527-2.

Kallio U, Poutanen M (2012) Can we really promise a mm-accuracy for the local ties on a geo-VLBI antenna, In: Geodesy for Planet Earth. Proceedings of the 2009 IAG Symposium, Buenos Aires, Argentina, 31 August 31-4 September 2009, Eds. Kenyon, S; M. Pacino; U. Marti. International Association of Geodesy Symposia, 136, 35-42, Springer Verlag, doi:10.1007/978-3-642- 20338-1_5.

Schmid R, Steigenberger P, Gendt G, Ge M, Rothacher M (2007) Generation of a consistent absolute phase center correction model for GPS receiver and satellite antennas, J. Geod., 81, 781-798, doi:10.1007/s00190-007-0148-y.

Snajdrova K, Boehm J, Willis P, Haas R, Schuh H (2005) Multi-technique comparison of tropospheric zenith delays derived during the CONT02 campaign, J. Geod., 79, 613-623, doi:10.1007/s00190-005-0010-z.

Teunissen PJG (1993) Least-squares estimation of the integer GPS ambiguities. IAG General Meeting, Invited Lecture, Section IV: Theory and methodology, Beijing, China. 
Webb FH, Zumberge JF (1993) An Introduction to the GIPSY/OASIS-II, JPL Publ. D-11088, Jet Propulsion Laboratory, Pasadena, California.

460 Zumberge JF, Heflin MB, Jefferson DC, Watkins MM, Webb FH (1997) Precise Point

461 Positioning for the Efficient and Robust Analysis of GPS Data from Large Networks, J. Geophys. Res., 102 (B3), 5005-5017, doi:10.1029/96JB03860. 

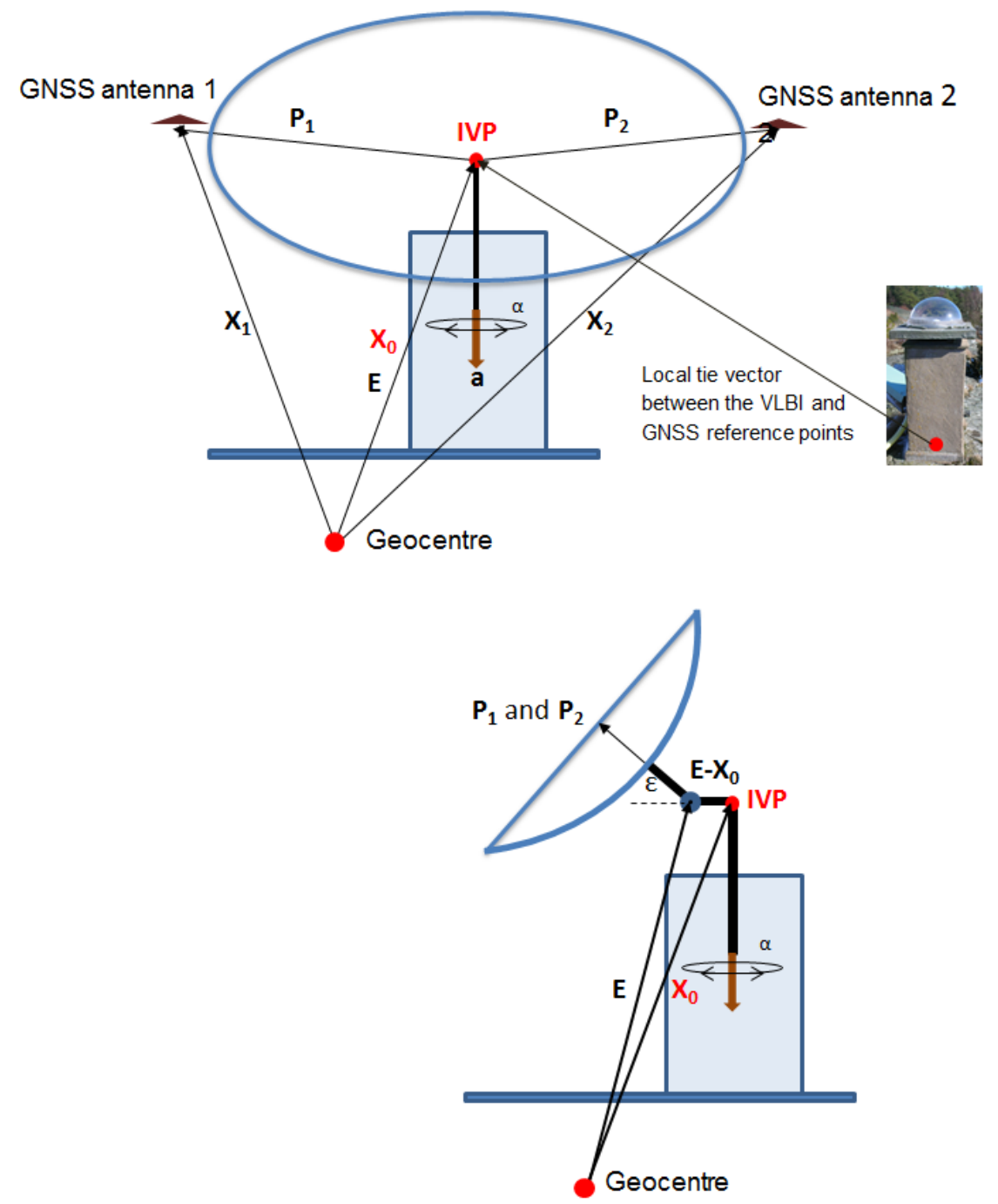

Fig. 1 A sketch of the model parameters and the local tie vector between the VLBI and the GNSS reference points illustrating the vectors involved (top). Ideally the two vectors $E$ and $X_{0}$ shall be identical. This is, however, not the case, which is illustrated in the bottom sketch and further described in the text. 
(a)
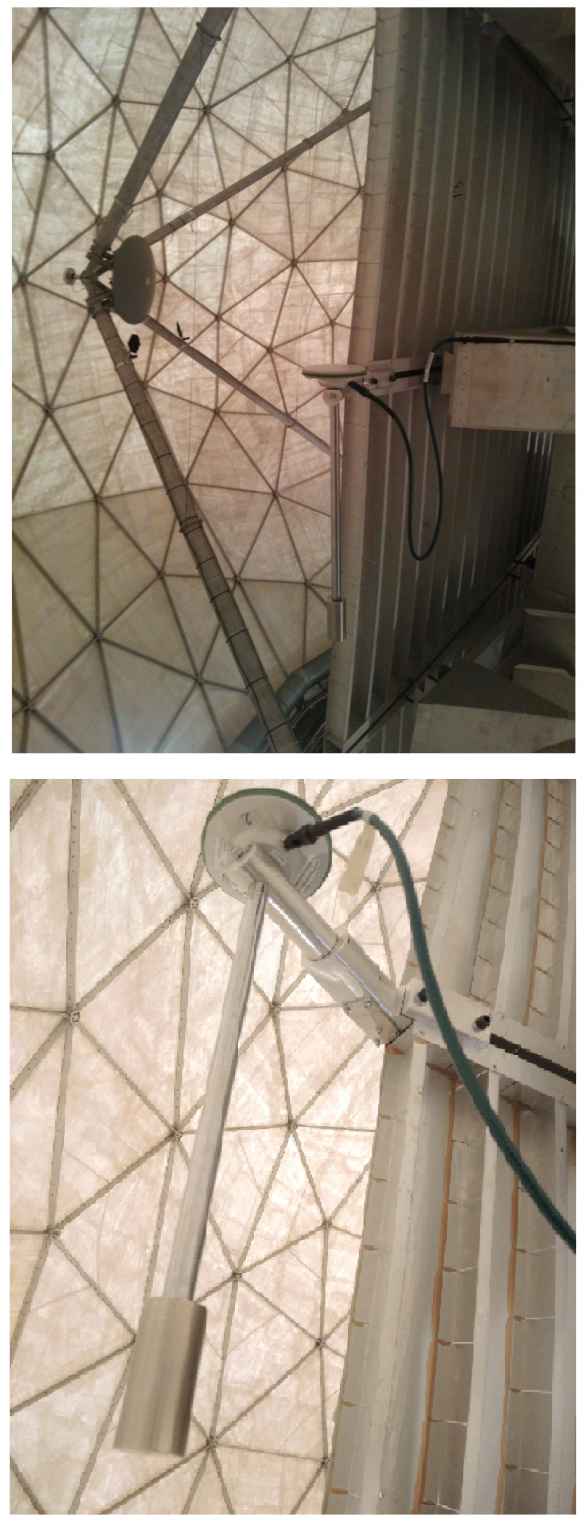

(b)
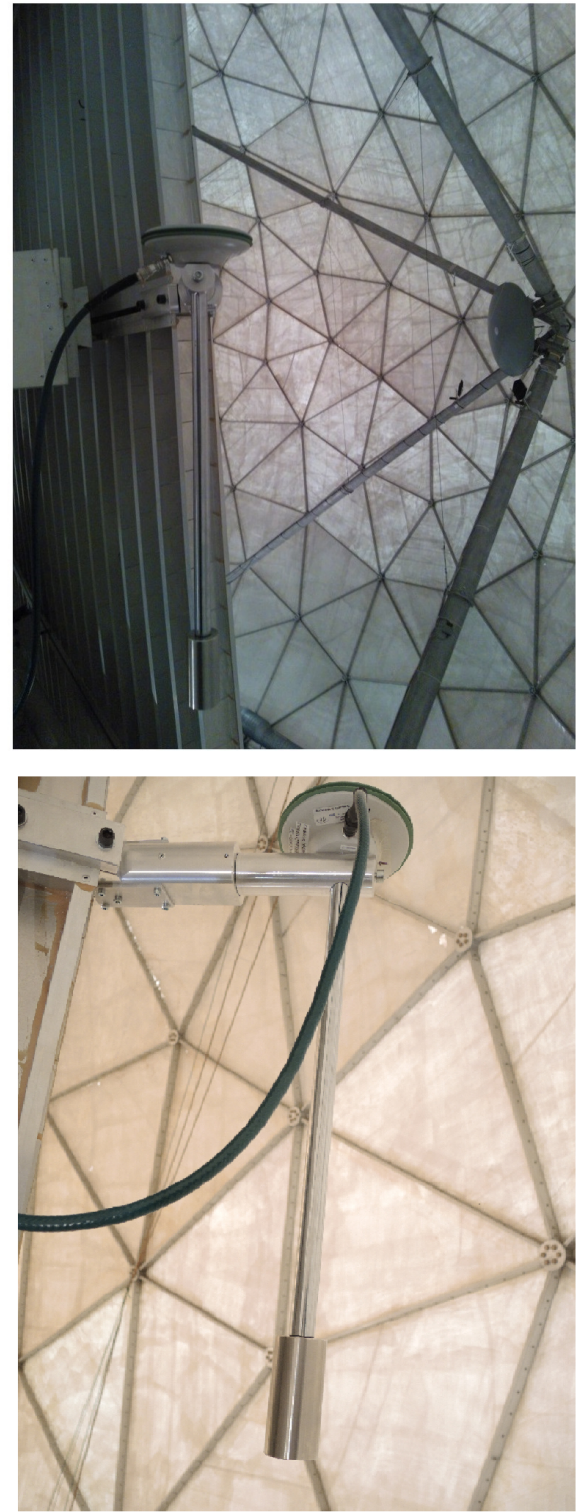

Fig. 2 The installation of the GNSS antennas on (a) the left side and (b) the right side of the $20 \mathrm{~m}$ radio telescope. The figures at the bottom show a close look of the two antennas. 


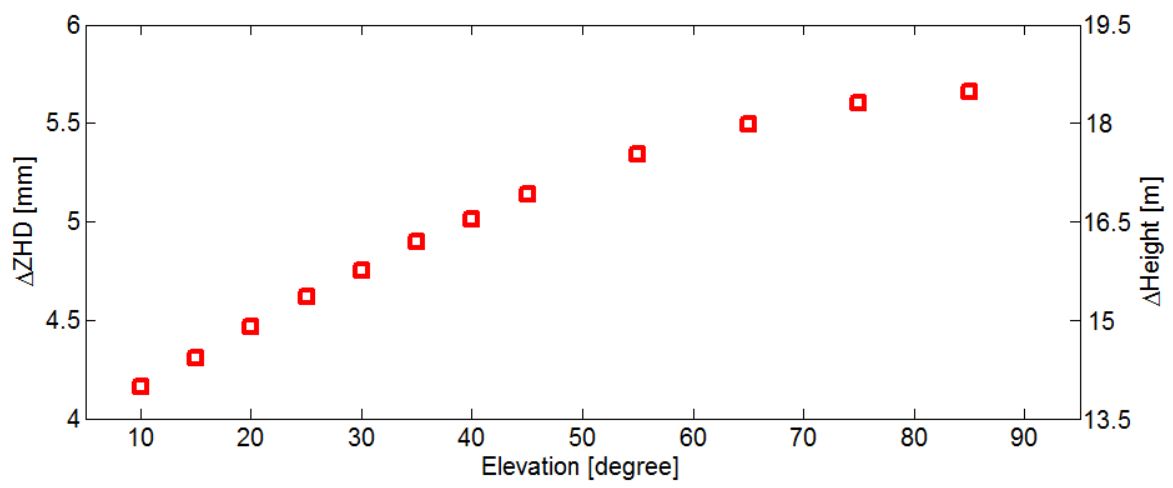

Fig. 3 The difference of the Zenith Hydrostatic Delay (ZHD) due to the height difference between the two GNSS antennas on the telescope and ONSA for different elevations of the telescope. The relation is given by the equation: $\triangle \mathrm{ZHD}=0.0003 * \triangle$ Height (Snajdrova et al., 2005).
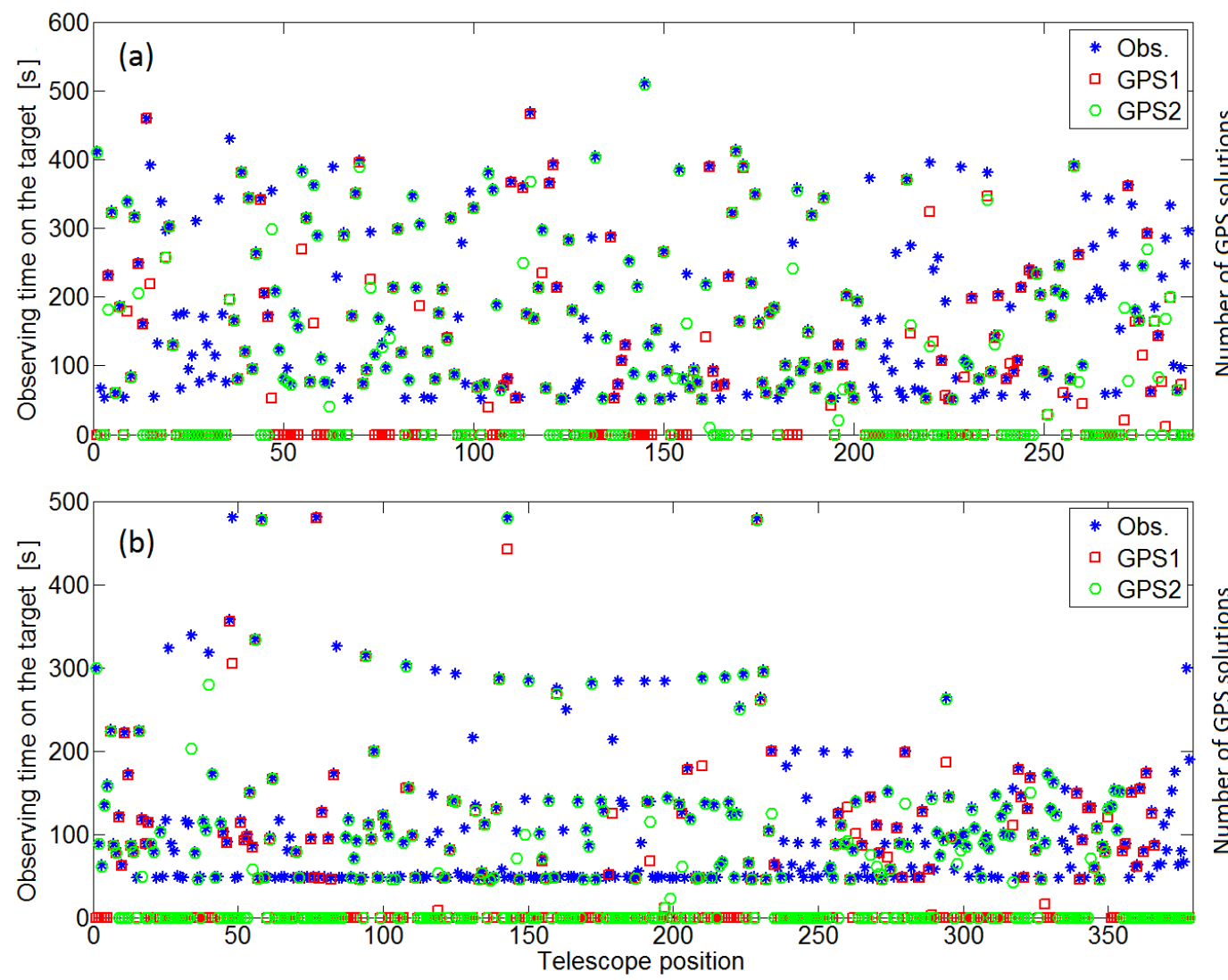

Fig. 4 The length of the observing time (left scale) while the telescope was tracking on a target at each position and the corresponding number of GPS solutions (one solution each second) for GPS1 and GPS2 (right scale) shown for the two kinematic sessions: (a) R1592 and (b) RV101. 
Table 1 Number of data points and telescope positions.

\begin{tabular}{|c|c|c|c|c|c|c|c|c|}
\hline & \multirow[b]{2}{*}{ Session } & \multicolumn{3}{|c|}{ No. of data points } & \multicolumn{4}{|c|}{ No. of telescope positions } \\
\hline & & Step $1^{1}$ & Step $2^{2}$ & Step $3^{3}$ & Step $0^{4}$ & Step 1 & Step 2 & Step 3 \\
\hline & semi-kinematic & & & & & & & \\
\hline 1 & 2013/07/09 & 65948 & 28260 & 27491 & 48 & 41 & 40 & 38 \\
\hline 2 & $2013 / 07 / 10$ & 66149 & 25964 & 25063 & 48 & 37 & 37 & 32 \\
\hline 3 & $2013 / 09 / 21$ & 60765 & 31717 & 30531 & 96 & 87 & 73 & 63 \\
\hline 4 & $2013 / 09 / 22$ & 60982 & 29085 & 27471 & 96 & 80 & 69 & 57 \\
\hline \multirow[t]{2}{*}{5} & $2013 / 09 / 23$ & 60336 & 27468 & 22833 & 96 & 76 & 70 & 57 \\
\hline & kinematic & & & & & & & \\
\hline 6 & R1592 (2013/07/01-07/02) & 26588 & 13738 & 13140 & 288 & 112 & 64 & 60 \\
\hline 7 & EUR124 (2013/07/04-07/05) & 28502 & 13704 & 13111 & 240 & 108 & 55 & 53 \\
\hline 8 & RV101 (2013/09/11-09/12) & 16662 & 3604 & 3190 & 378 & 93 & 22 & 19 \\
\hline 9 & R1604 (2013/09/24-09/25) & 25816 & 8484 & 8043 & 253 & 81 & 24 & 22 \\
\hline
\end{tabular}

1 After the GPS data processing.

${ }^{2}$ After the outlier exclusion.

${ }^{3}$ The last iteration in the least squares mixed model.

${ }^{4}$ The total numbers of positions that the telescope was positioned at in each session. 
Table 2 The estimated local tie vector between the VLBI and the GNSS reference points, and the estimated axis offset of the telescope as well as the estimated P vectors.

\begin{tabular}{|c|c|c|c|c|c|c|c|c|}
\hline & Session Date & $\begin{array}{c}\triangle \mathrm{X}^{1} \\
{[\mathrm{~m}]}\end{array}$ & $\begin{array}{c}\triangle \mathrm{Y}^{1} \\
{[\mathrm{~m}]}\end{array}$ & $\begin{array}{c}\triangle \mathrm{Z}^{1} \\
{[\mathrm{~m}]}\end{array}$ & $\begin{array}{c}\text { Baseline } \\
{[\mathrm{m}]}\end{array}$ & $\begin{array}{c}\text { Axis offset } \\
{[\mathrm{m}]}\end{array}$ & $\begin{array}{l}\mathrm{P}_{1} \\
{[\mathrm{~m}]}\end{array}$ & $\begin{array}{l}\mathrm{P}_{2} \\
{[\mathrm{~m}]}\end{array}$ \\
\hline & \multicolumn{8}{|l|}{ semi-kinematic } \\
\hline 1 & 2013-07-09 & -52.6283 & 40.4624 & 43.8743 & 79.5732 & -0.0083 & 12.0615 & 12.0629 \\
\hline 2 & 2013-07-10 & -52.6277 & 40.4635 & 43.8741 & 79.5732 & -0.0057 & 12.0626 & 12.0613 \\
\hline 3 & 2013-09-21 & -52.6286 & 40.4638 & 43.8755 & 79.5747 & -0.0033 & 12.0617 & 12.0629 \\
\hline 4 & 2013-09-22 & -52.6295 & 40.4637 & 43.8745 & 79.5748 & -0.0071 & 12.0618 & 12.0624 \\
\hline \multirow[t]{2}{*}{5} & 2013-09-23 & -52.6296 & 40.4637 & 43.8764 & 79.5759 & -0.0063 & 12.0615 & 12.0615 \\
\hline & Mean & -52.6287 & 40.4634 & 43.8749 & 79.5744 & -0.0061 & 12.0618 & 12.0622 \\
\hline & Standard deviation & 0.0008 & 0.0006 & 0.0010 & 0.0011 & 0.0019 & 0.0005 & 0.0008 \\
\hline & \multicolumn{8}{|l|}{ kinematic } \\
\hline 6 & R1592 (2013/07/01-07/02) & -52.6273 & 40.4647 & 43.8764 & 79.5749 & -0.0050 & 12.0648 & 12.0621 \\
\hline 7 & EUR124 (2013/07/04-07/05) & -52.6283 & 40.4643 & 43.8740 & 79.5740 & -0.0096 & 12.0655 & 12.0612 \\
\hline 8 & RV101 (2013/09/11-09/12) & -52.6323 & 40.4623 & 43.8665 & 79.5715 & -0.0069 & 12.0659 & 12.0620 \\
\hline \multirow[t]{9}{*}{9} & R1604 (2013/09/24-09/25) & -52.6290 & 40.4653 & 43.8741 & 79.5750 & -0.0052 & 12.0611 & 12.0590 \\
\hline & Mean & -52.6292 & 40.4642 & 43.8728 & 79.5738 & -0.0067 & 12.0643 & 12.0610 \\
\hline & Standard deviation & 0.0022 & 0.0013 & 0.0043 & 0.0016 & 0.0021 & 0.0022 & 0.0014 \\
\hline & Mean (total) & -52.6290 & 40.4638 & 43.8740 & 79.5741 & -0.0064 & 12.0629 & 12.0617 \\
\hline & Standard deviation (total) & 0.0015 & 0.0010 & 0.0029 & 0.0013 & 0.0019 & 0.0019 & 0.0012 \\
\hline & ITRF2008 & -52.6270 & 40.4650 & 43.8690 & 79.5708 & & & \\
\hline & Difference from ITRF2008 & 0.0020 & -0.0012 & 0.0050 & 0.0033 & & & \\
\hline & Local survey 2002 & & & & $79.5685^{2}$ & -0.0060 & & \\
\hline & Local survey 2008 & & & & $79.5678^{2}$ & -0.0062 & & \\
\hline
\end{tabular}

${ }^{1}$ The vector is defined by VLBI-GNSS.

${ }^{2}$ Taken from Table 3 in Lösler and Haas (2009) where all baselines were calculated referring to a temperature of $0{ }^{\circ} \mathrm{C}$.

Table 3 Covariance matrix for the local tie vector between the VLBI and the GNSS reference points. The units are $\mathrm{mm}^{2}$

\begin{tabular}{llll}
\hline & $\triangle X$ & $\triangle Y$ & $\triangle Z$ \\
$\triangle X$ & 3.99 & 0.10 & 3.83 \\
$\triangle Y$ & 0.10 & 0.59 & 0.03 \\
$\triangle \mathrm{Z}$ & 3.83 & 0.03 & 5.96 \\
\hline
\end{tabular}


Table 4 The same results as in Table 2, but here the local tie vector is given in topocentric coordinates.

\begin{tabular}{|c|c|c|c|c|}
\hline & Session Date & $\begin{array}{c}\text { East }^{1} \\
{[\mathrm{~m}]}\end{array}$ & $\begin{array}{c}\text { North }^{1} \\
{[\mathrm{~m}]}\end{array}$ & $\begin{array}{c}\text { Vertical }^{1} \\
{[\mathrm{~m}]}\end{array}$ \\
\hline & \multicolumn{4}{|l|}{ semi-kinematic } \\
\hline 1 & 2013-07-09 & 50.4642 & 59.9754 & 13.7192 \\
\hline 2 & 2013-07-10 & 50.4652 & 59.9746 & 13.7195 \\
\hline 3 & 2013-09-21 & 50.4656 & 59.9760 & 13.7202 \\
\hline 4 & 2013-09-22 & 50.4658 & 59.9762 & 13.7189 \\
\hline \multirow[t]{4}{*}{5} & $2013-09-23$ & 50.4658 & 59.9773 & 13.7204 \\
\hline & Mean & 50.4653 & 59.9759 & 13.7197 \\
\hline & Standard deviation & 0.0007 & 0.0010 & $\mathbf{0 . 0 0 0 7}$ \\
\hline & \multicolumn{4}{|l|}{ kinematic } \\
\hline 6 & R1592 (2013/07/01-07/02) & 50.4663 & 59.9753 & 13.7218 \\
\hline 7 & EUR124 (2013/07/04-07/05) & 50.4661 & 59.9749 & 13.7193 \\
\hline 8 & RV101 (2013/09/11-09/12) & 50.4649 & 59.9745 & 13.7105 \\
\hline \multirow[t]{7}{*}{9} & R1604 (2013/09/24-09/25) & 50.4672 & 59.9753 & 13.7190 \\
\hline & Mean & 50.4661 & 59.9750 & 13.7176 \\
\hline & Standard deviation & 0.0009 & 0.0004 & 0.0049 \\
\hline & Mean (total) & 50.4657 & 59.9754 & 13.7188 \\
\hline & Standard deviation (total) & 0.0008 & 0.0009 & $\mathbf{0 . 0 0 3 2}$ \\
\hline & ITRF2008 & 50.4665 & 59.9710 & 13.7157 \\
\hline & Difference from ITRF2008 & -0.0008 & 0.0044 & 0.0031 \\
\hline
\end{tabular}

${ }^{1}$ The vector is defined by VLBI-GNSS. 
Fig. 5 The estimated local tie vector and the axis offset of the telescope obtained from each session. The results are given by the GPS data processing with (red circles) and without (blue squares) fixing the baseline between the two GNSS antennas on the VLBI telescope. The session number is given in Tables 1 and 2. The calculated ITRF2008 local tie vectors are given by black solid lines while the line for the axis offset was obtained using the mean value of the two local surveys.
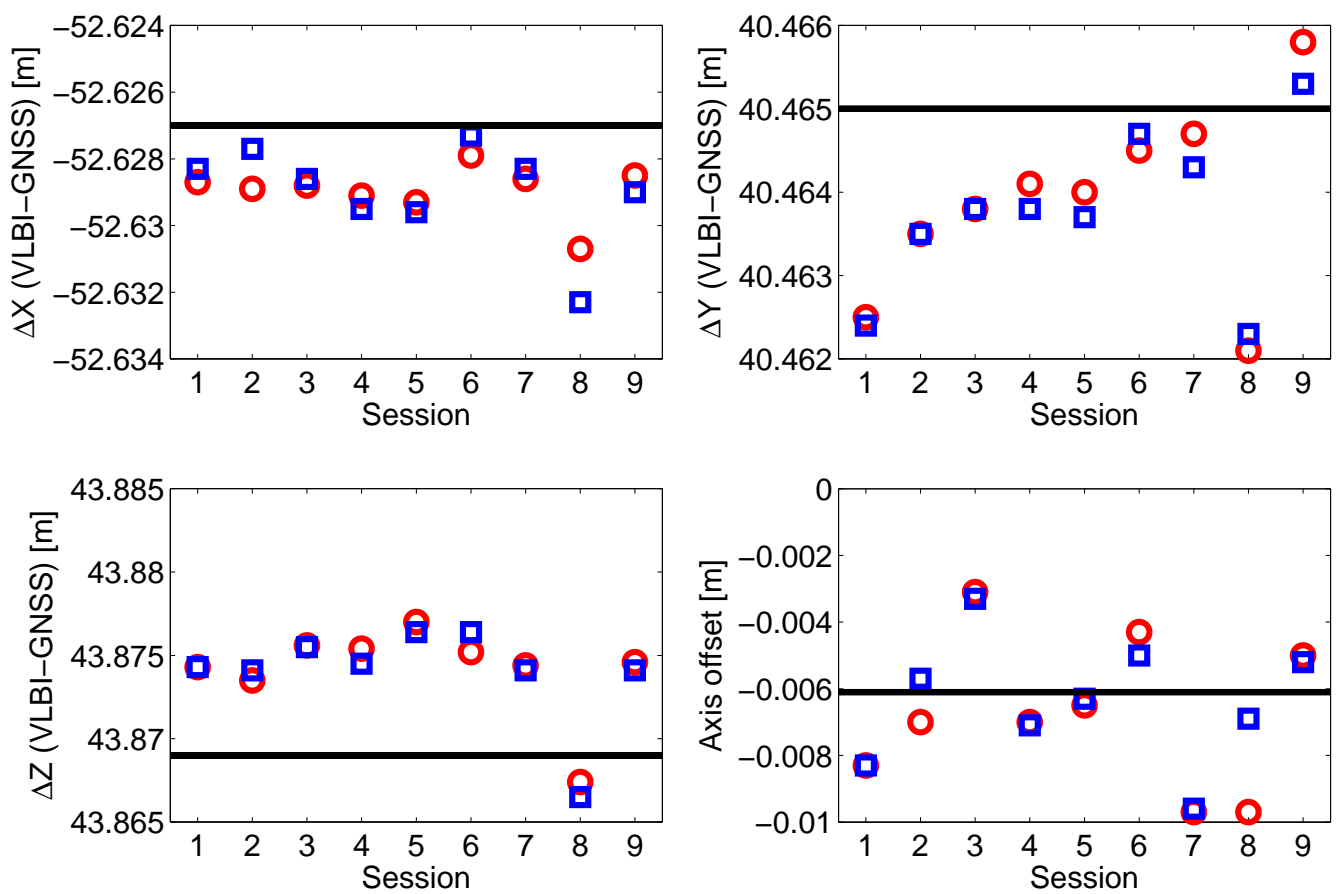
Fig. 6 The estimated local tie vector from each session with (blue crosses) and without (red circles) fixing the axis offset of the telescope. The fixed axis offset value is $-6.1 \mathrm{~mm}$ (the mean value of axis offset obtained by the two local surveys (Lösler and Haas, 2009)). The session number is given in Tables 1 and 2 while the calculated ITRF2008 local tie vectors are given by black solid lines.

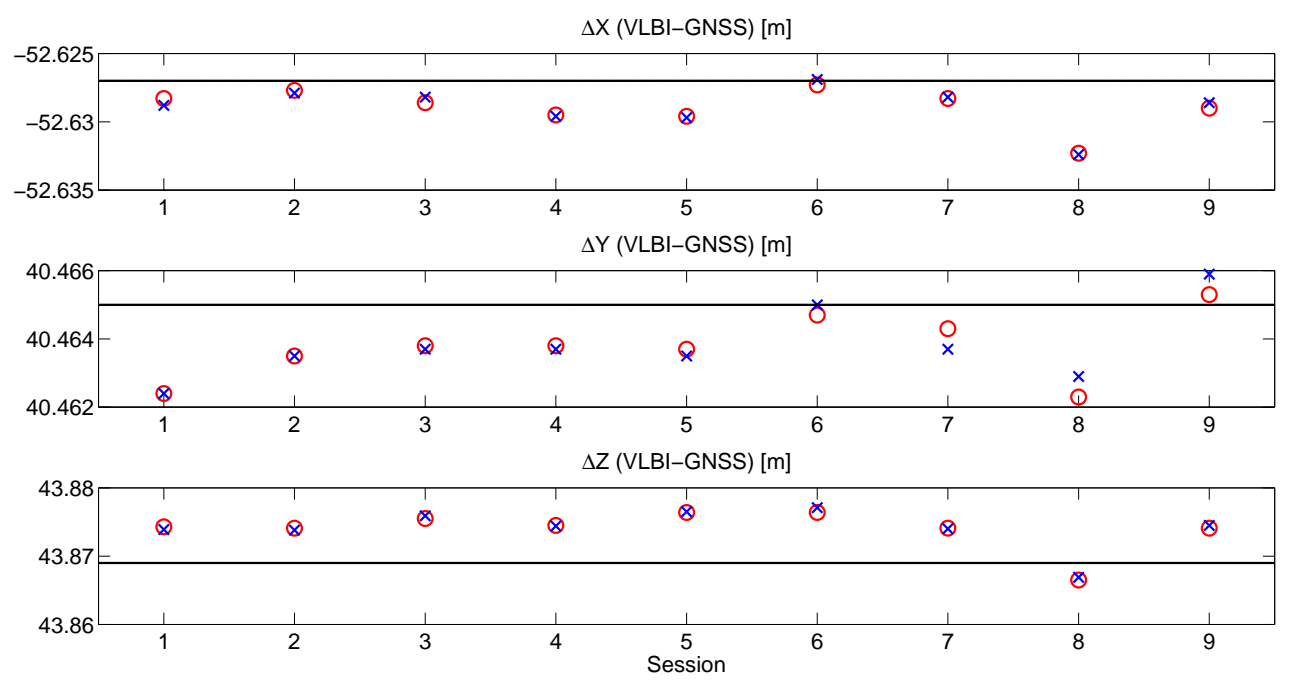


Fig. 7 The estimated local tie vector and the axis offset of the telescope obtained from each session. The blue squares show the results given by the GPS data processing using the standard absolute PCV corrections and the red circles show the results using the PCV corrections which were calculated based on the azimuth orientation of the telescope. The session number is given in Tables 1 and 2. The calculated ITRF2008 local tie vectors are given by black solid lines while the line for the axis offset was obtained using the mean value of the two local surveys.
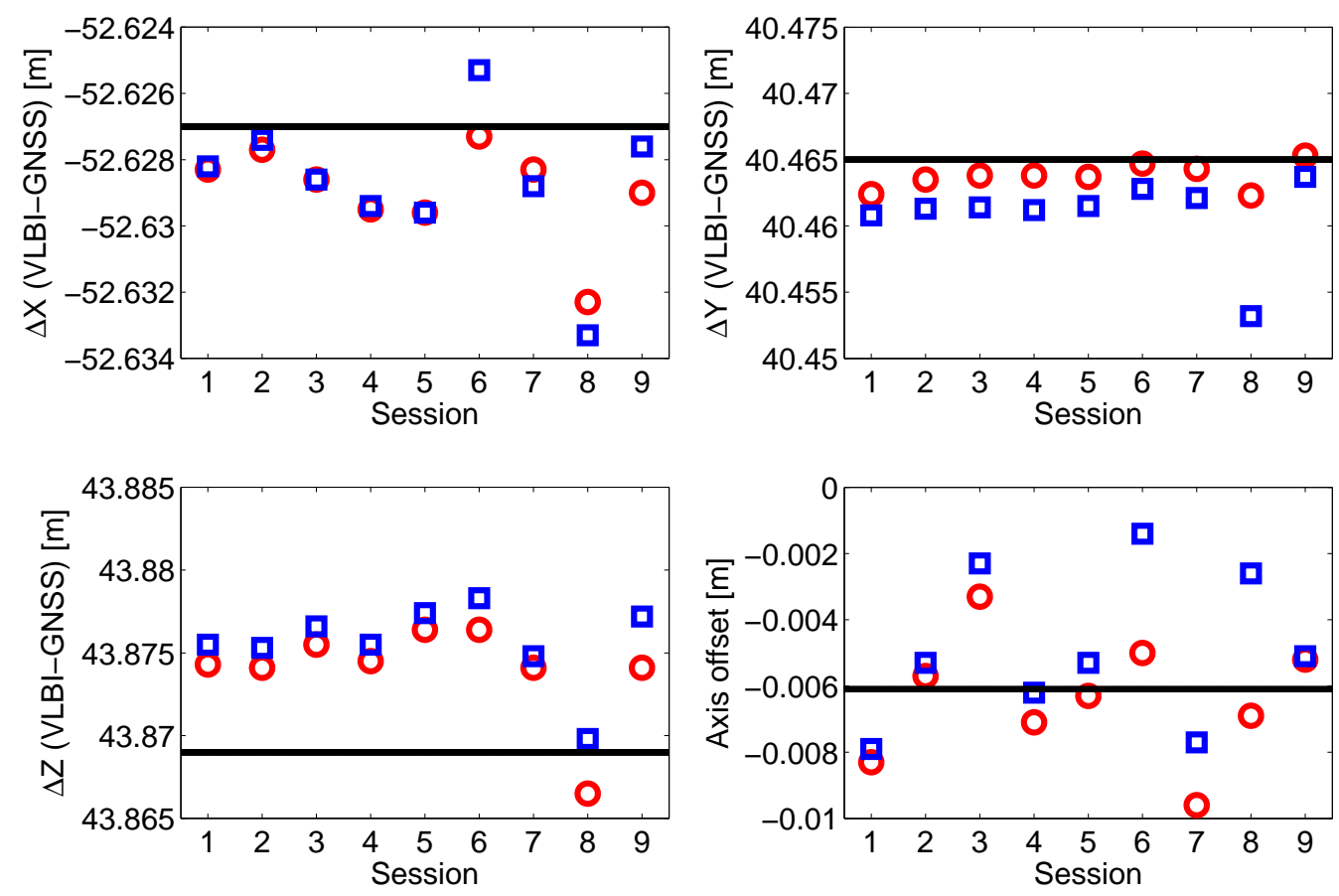